\title{
Extreme obesity induces massive beta cell expansion in mice through self-renewal and does not alter the beta cell lineage
}

\author{
Aaron R. Cox ${ }^{1,2}$ • Carol J. Lam ${ }^{1,2} \cdot$ Matthew M. Rankin ${ }^{3} \cdot$ Kourtney A. King ${ }^{3}$ • \\ Pan Chen ${ }^{3}$ - Ramon Martinez ${ }^{1,2}$ - Changhong $\mathrm{Li}^{3}$ - Jake A. Kushner ${ }^{1,2,4}$
}

Received: 8 December 2015 / Accepted: 22 February 2016 / Published online: 22 March 2016

(C) The Author(s) 2016. This article is published with open access at Springerlink.com

\begin{abstract}
Aims/hypothesis Understanding the developmental biology of beta cell regeneration is critical for developing new diabetes therapies. Obesity is a potent but poorly understood stimulus for beta cell expansion. Current models of obesity are complicated by developmental compensation or concurrent diabetes, limiting their usefulness for identifying the lineage mechanism(s) of beta cell expansion. We aimed to determine whether acute inducible obesity stimulates beta cell expansion and to determine the lineage mechanism of beta cell growth in obesity. Methods We created whole-body tamoxifen-inducible leptin receptor ( LepR)-deficient mice $\left(U b c-C r e^{E R T 2} L e p R^{\text {loxP/loxP }}\right)$ as a novel model of acute obesity. Beta cell mass and proliferation were quantified after short-term LepR deletion. Clonal analysis of beta cell expansion using the Brainbow2.1 reporter was performed 6 months post tamoxifen initiation.

Results LepR deficiency induced a doubling of body mass within 3 weeks, with moderate glucose intolerance (unlike
\end{abstract}

Electronic supplementary material The online version of this article (doi:10.1007/s00125-016-3922-7) contains peer-reviewed but unedited supplementary material, which is available to authorised users.

Jake A. Kushner

kushner@bcm.edu

1 Section of Pediatric Diabetes and Endocrinology, McNair Medical Institute, Baylor College of Medicine, Houston, TX 77030, USA

2 Diabetes and Endocrinology, Feigin Center, Texas Children's Hospital, Houston, TX, USA

3 Division of Endocrinology and Diabetes, Children's Hospital of Philadelphia, Perelman School of Medicine, University of Pennsylvania, Philadelphia, PA, USA

4 Diabetes and Endocrinology Service, Texas Children's Hospital, Houston, TX, USA typical LepR mutant mice [ $d b / d b]$, which have frank diabetes). Beta cell mass expanded threefold through increased beta cell proliferation, without evidence for contribution from specialised progenitors or stem cells (via sequential thymidine labelling and Brainbow2.1 reporter). Thus, self-renewal is the primary lineage mechanism in obesity-induced beta cell expansion. However, even the rapid beta cell proliferation could not exceed the restrictions of the replication refractory period. Conclusions/interpretation In summary, we created a novel model of inducible obesity demonstrating that even extreme metabolic demand does not alter beta cell lineage.

Keywords Beta cell expansion $\cdot$ Cell fate $\cdot$ Lineage tracing . Obesity
Abbreviations
BrdU 5-Bromo-2'-deoxyuridine
CFP Cyano-fluorescent protein
EdU 5-Ethynyl-2'-deoxyuridine
ER Endoplasmic reticulum
GFP Green fluorescent protein
HFD High-fat diet
ITT Insulin tolerance test
KO Knockout
LepR Leptin receptor
PPx Partial pancreatectomy
RFP Red fluorescent protein
YFP Yellow fluorescent protein

\section{Introduction}

Absolute or relative deficiency of beta cell mass underlies diabetes. Endogenous beta cell regeneration represents a 
promising approach to restore functional beta cell mass. However, adult beta cell turnover is minimal, with very low rates of beta cell proliferation and apoptosis [1-4]. Current efforts to stimulate human beta cell regeneration have proven futile [5-9], although obese individuals have profound beta cell expansion compared with lean individuals $[4,10]$. Thus, obesity represents an exceptional model with which to study the mechanisms of beta cell regeneration.

Very little is known about the lineage mechanism of beta cell expansion in obesity. Butler et al observed an increase in insulin-positive cells associated with exocrine ducts [10]. Given the low rates of beta cell turnover observed in obese humans, they proposed that obesity-induced beta cell growth occurred by neogenesis through duct cell differentiation. However, the inherent limitations of post-mortem human samples prevent lineage tracing.

Various rodent models have demonstrated that adult beta cell growth and regeneration occur primarily by selfduplication [11-16]. However, metabolic demand is one of the most powerful stimuli for beta cell mass expansion [10, $17,18]$ and thus obesity might alter beta cell fate through recruitment of specialised progenitors.

Several shortcomings limit current rodent models for the study of obesity-induced beta cell expansion. Long-term administration of a high-fat diet (HFD) can produce highly variable results [19] and beta cell expansion can take several weeks to occur [20]. Constitutive leptin receptor (LepR) signalling mutant models ( $d b / d b$ and $o b / o b$ mice, $f a / f a$ rats) develop obesity through germ line loss of leptin signalling. Reduced leptin signalling throughout embryonic development may provoke compensatory changes that limit the study of postnatal beta cells. These mutant rodents develop frank diabetes early in life, further complicating the study of beta cell turnover. An acute model of obesity is necessary to overcome these potential limitations. We developed a novel model of acute obesity to definitively clarify the lineage mechanism of beta cell mass expansion in obesity.

\section{Methods}

Mice Experiments were performed at Baylor College of Medicine and Children's Hospital of Philadelphia according to Institutional Animal Care and Use Committee protocols. Rosa26(CAG-Brainbow2.1) (JAX no. 013731), Rosa26 $6^{(\text {loxP-stop-loxP-EYFP) }}$ (JAX no. 006148) and LepR RoxPloxP (JAX no. 008327) [21] mice were obtained from Jackson (Bar Harbor, ME, USA). Ubc-Cre ${ }^{E R T 2}$ mice were from E. Brown at the University of Pennsylvania [22]. Crosses yielded $U b c$ Cre ${ }^{E R T 2}$ Rosa $26^{E Y F P} L_{\text {epR }}^{\text {loxP/loxP }}$ and Ubc-Cre $e^{E R T 2}$ Rosa26 ${ }^{(\text {CAG-Brainbow2.1) }}$ LepR $^{\text {loxPloxP }}$ mice on a B6.129 F1 hybrid background, genotyped with REDExtract-N-Amp (SigmaAldrich, St Louis, MO, USA) (ESM Table 1). Male and female mice (5-6 weeks of age) were gavaged with tamoxifen $(0.1 \mathrm{mg} / \mathrm{g}$; MP, Santa Ana, CA, USA) for 5 days. Mice were labelled via drinking water with 5-bromo-2'-deoxyuridine (BrdU; $1 \mathrm{~g} /$ l; Sigma-Aldrich) or 5-ethynyl-2'-deoxyuridine (EdU; 0.5 g/l; Life Technologies, Grand Island, NY, USA), as described previously [23]. Intraperitoneal GTTs were performed as described previously [16]. Insulin tolerance tests (ITTs) were performed after $4 \mathrm{~h}$ fasting, using human regular insulin (1 U/kg; Eli Lilly, Indianapolis, IN, USA). Serum insulin was measured using a Mouse Ultrasensitive Insulin ELISA (Alpco Diagnostics, Salem, NH, USA). Mice were fed an HFD (60\% of energy from fat; D12492; Research Diets, New Brunswick, NJ, USA) or chow diet ( $22 \%$ of energy from fat; No. 2919; Harlan, Houston, TX, USA). Randomisation of groups was not possible given the overt phenotype.

Gene deletion gDNA was extracted using Quick-gDNA MiniPrep (Zymo Research, Irvine, CA, USA). LepR gene deletion was assessed via Sybr Green (Sigma-Aldrich) qPCR (for primers see ESM Table 1).

In vitro islet function Islets isolated from individual mice at 1 week were cultured in RPMI 1640 medium with $10 \mathrm{mmol} / \mathrm{l}$ glucose and $10 \%$ fetal bovine serum for 2 days. Islet function was evaluated by perifusion as previously [24], with $3 \mathrm{mmol} / 1$ basal glucose (ramp of $0.625 \mathrm{mmol} \mathrm{l}^{-1} \mathrm{~min}^{-1}$ ), followed by $30 \mathrm{mmol} / \mathrm{K} \mathrm{KCl}$ stimulation at completion. Insulin secretion was measured by HTRF assay (Cisbio, Bedford, MA, USA). Cytosolic calcium was measured as described previously [24]. Fura-2AM (Life Technologies) was used as a calcium indicator and was measured with a Zeiss AxioVision microscope (Carl Zeiss, Thornwood, NY, USA).

Immunohistochemistry and morphometry Paraffin sections were prepared as described previously [23]. Primary antisera included guinea pig anti-insulin (Dako, Carpinteria, CA, USA) and rat anti-BrdU (Accurate Chemical, Westbury, NY, USA), followed by secondary antisera conjugated to $\mathrm{Cy} 2 / \mathrm{Cy} 3$ (Jackson ImmunoResearch, West Grove, PA, USA) and DAPI (Molecular Probes, Eugene, OR, USA). EdU was detected using Click-iT EdU Alexa Fluor 647 (Life Technologies) according to the manufacturer's protocol. Slides were imaged to quantify beta cell morphometry as described previously [25], using Volocity 6.1.1 (PerkinElmer, Waltham, MA, USA). BrdU-positive, EdU-positive and BrdU/EdU co-positive beta cell ratios to total beta cells were calculated, and the percentage of predicted co-positive cells was obtained by dividing the percentage of actual co-positive cells by the percentage of predicted co-positive cells, multiplied by $100 \%$. At least 3,000 beta cells were counted per mouse. Blinding of samples was not possible given the overt phenotype. 
Clonal analysis Brainbow2.1 (Rosa26(CAG-Brainbow2.1) $)$ expresses one of four colours (cyano-fluorescent protein [CFP], red fluorescent protein [RFP], yellow fluorescent protein [YFP] or green fluorescent protein [GFP]) from each allele [26, 27]. We were unable to reliably separate the spectral overlap for YFP and GFP, thus these were grouped together and represented as green in all images. Contiguous clones were identified as insulinpositive cells expressing a fluorescent protein(s) with a common border. Cells/clone were counted, with at least 230 clones counted per sample.

Statistics Results were reported as mean \pm SEM unless noted otherwise, and compared with independent Student's $t$ tests (unpaired). No samples have been excluded from analysis.

\section{Results}

Acute $L e p R$ deficiency results in massive, progressive weight gain with moderate changes in glucose homeostasis We created a unique model of obesity by deriving whole-body LepR-deficient (Ubc-Cre $e^{E R T 2} L e p R^{\text {loxP/loxP }}$; LepR-knockout [KO]) mice on a B6.129 F1 hybrid background, resulting in tamoxifen-induced LepR gene deletion (ESM Fig. 1). We characterised the metabolic phenotype of LepR-KO and $L_{e p R} R^{\text {loxP/loxP }}$ littermate controls (Fig. 1a). Both sexes demonstrated equivalent phenotypical characteristics following LepR deletion (ESM Tables 2, 3). LepR-KO mice displayed extreme weight gain, with their weight having increased by more than twofold by week 5 (Fig. 1b; control $17.8 \mathrm{~g}$ vs LepR-KO $41.7 \mathrm{~g} ; p<0.0001)$. Random fed blood glucose levels transiently increased in LepR-KO mice, returning to control values by 5 weeks (Fig. 1c). Glucose tolerance gradually declined in LepR-KO mice, with significantly elevated fasting blood glucose concentrations (Fig. 1d-f). Insulin resistance developed in LepR-KO mice within 1 week (Fig. 1g, h). Thus, acute LepR deletion leads to extreme obesity with moderate changes in glucose homeostasis.

LepR-KO mice do not develop frank diabetes by compensating, in part, through increased beta cell function. Fed and fasting serum insulin levels were increased in LepR-KO mice compared with controls (Fig. 1i, j); measurement of basal and glucose-stimulated insulin secretion suggested that LepR-KO islets secreted less insulin despite a $41 \%$ increase in total insulin content compared with controls (Fig. 1k-n). Glucose stimulation index was preserved in LepR-KO islets (Fig. 11), as was stimulated calcium release (Fig. 1o-q). Importantly, LepR-KO islets remain responsive to glucose, despite blunted basal and stimulated insulin release. Thus, islet dysfunction did not confound our ability to analyse beta cell mass expansion in response to acute obesity.
Acute $L e p R$ deficiency results in massive, rapid beta cell mass expansion We hypothesised that beta cells expand in response to extreme obesity to meet the increasing insulin demand. We measured beta cell morphometry at various times after tamoxifen initiation (Fig. 2a). Beta cell mass increased by more than twofold in LepR-KO mice compared with control mice at 3 weeks and was further increased at 5 weeks (Fig. 2b-d and ESM Table 4). Beta cell size and crosssectional beta cell area per islet were greater in LepR-KO mice and there were more large islets (ESM Table 5). Thus, obesity associated with acute LepR deficiency induced massive, rapid beta cell expansion.

Acute LepR deficiency rapidly and massively increases beta cell proliferation To examine the impact of acute obesity upon beta cell proliferation, we administered thymidine analogues for 2 weeks before mice were euthanised (Fig. 3a). Two-week thymidine incorporation in beta cells of control mice was $11 \%$ at 3 weeks and declined to $5 \%$ at 5 weeks (Fig. 3b, c and ESM Table 6). Beta cell proliferation dramatically increased to $70 \%$ in LepR-KO mice at 3 weeks. Despite continued metabolic demand at 5 weeks (Fig. 1), beta cell proliferation declined to 34\% (Fig. 3b, c and ESM Table 6). Notably, acute LepR deficiency represents one of the strongest known stimuli for beta cell replication.

Beta cells expand by self-renewal in acute LepR-deficient mice We tested if beta cells expand in obesity via highly proliferative 'transit amplifying' progenitors, as observed in skin or intestine. To determine the lineage mechanism of beta cell expansion, we employed sequential administration of two thymidine analogues (Fig.4a), as described previously [14, 16]. By labelling the first cell division with BrdU (green) and the second with EdU (red), sequential cell division resulted in BrdU/EdU co-labelled cells (Fig. 3b inset, Fig. 4a-c, [14]). If beta cells expand by specialised progenitors undergoing sequential cell division (transit amplifying population), then the beta cells would be BrdU/EdU double-positive (Fig. 4b). Alternatively, if beta cells expand by self-renewal through random cell division, few double-positive beta cells would be expected after obesity (Fig. 4c). During week 2 in control mice, $8 \%$ of beta cells incorporated BrdU while during week 3, 4\% of beta cells were labelled with EdU (Fig. 4d). BrdU/EdU co-positive beta cells were nearly absent in control mice (Fig. 4d, e and ESM Table 6), consistent with previous reports of beta cell expansion [14]. In contrast, acute LepR deletion resulted in labelling of $\sim 50 \%$ of beta cells in week 2 and $23 \%$ in week 3 . Despite substantial beta cell proliferation in LepR-KO mice, only a tiny fraction of beta cells were BrdU/EdU co-positive (2.3\%). Beta cell proliferation declined during weeks 4 and 5 in LepR-KO mice, but remained significantly elevated compared with controls, at 15-18\% (Fig. 4e). Accordingly, even fewer BrdU/EdU co-positive beta cells 


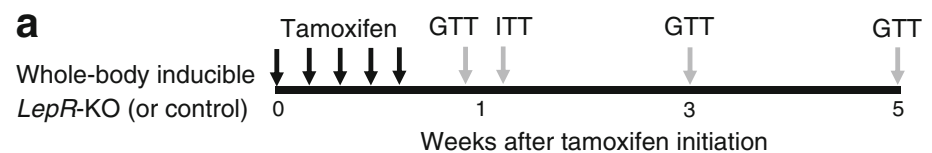

b

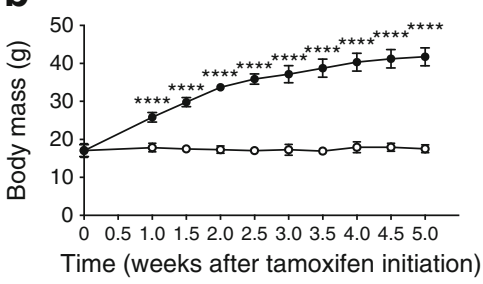

C

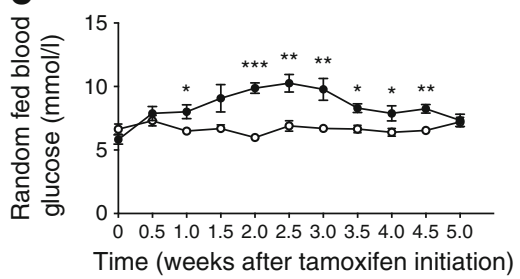

d

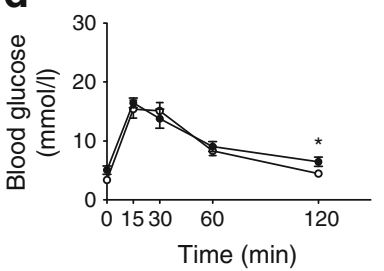

g

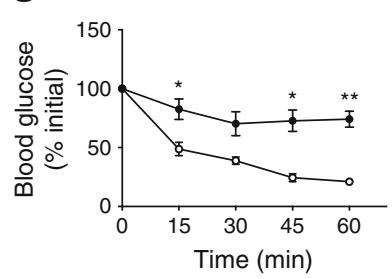

k

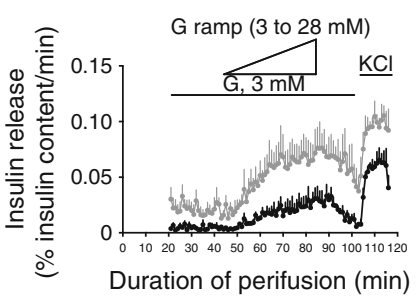

0
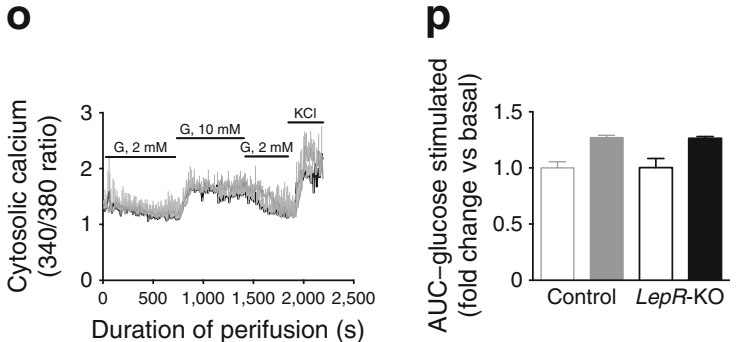

Fig. 1 Acute LepR deficiency in mice results in massive, progressive weight gain with moderate changes in glucose homeostasis. (a) Timing of LepR gene deletion, washout, ITTs and GTTs. (b, c) Body mass (b) and randomly fed blood glucose levels (c) measured twice a week. (d-f) GTTs performed at day 5 (d), week 3 (e) and week 5 (f). (g, h) ITTs performed at week $1(\mathbf{g})$; corresponding AUC is shown $(\mathbf{h})$. (i, j) Random fed (i) and fasting (j) serum insulin. White circles and bars, control; black circles and bars, LepR-KO. Data are means \pm SEM, except (b) (means $\pm \mathrm{SD}$ ); 3-7 mice per group. ${ }^{*} p<0.05,{ }^{*} p<0.01,{ }^{* * *} p<0.001$ and
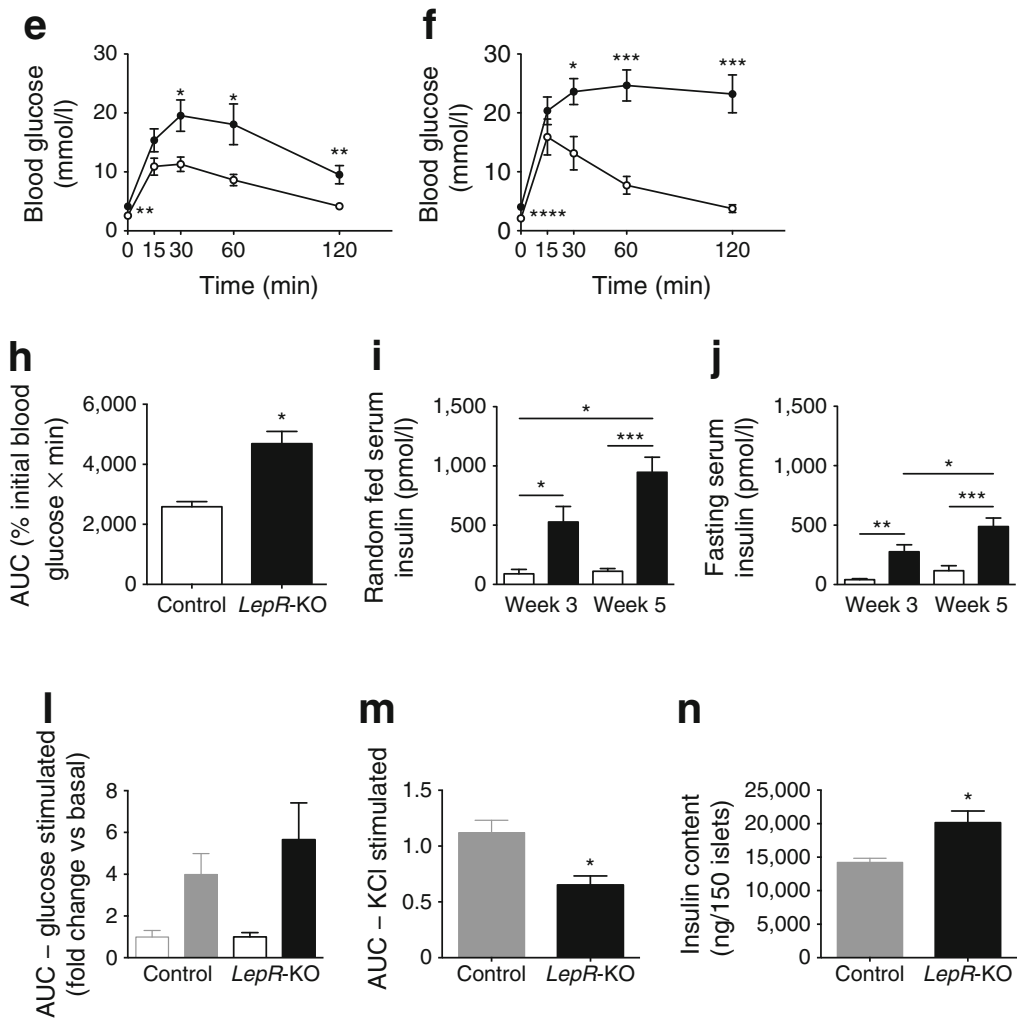

ก

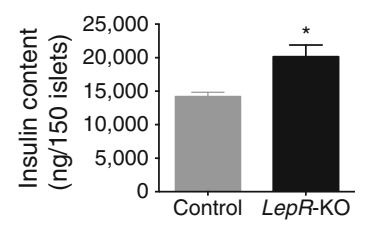

q

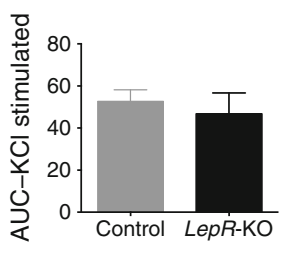

**** $p<0.0001$ vs control. (k-n) Insulin release from isolated islets with glucose (G) ramp, followed by $\mathrm{KCl}$ stimulation (k), with corresponding AUC for glucose (normalised to baseline/group) (l) and $\mathrm{KCl}$ simulation $(\mathbf{m})$, and total insulin content (n). (o-q) Cytosolic calcium release from isolated islets (o) and corresponding AUC for glucose (normalised to baseline/group) (p) and $\mathrm{KCl}$ stimulation (q). Grey traces, control; black traces, LepR-KO; white bars, basal; black/grey bars, stimulated. Data are means \pm SEM, 3 mice per group. ${ }^{*} p<0.05$ vs control 
Fig. 2 Acute LepR deficiency results in massive, rapid beta cell mass expansion. (a) Timing of LepR gene deletion and euthanasia (Euth). (b) Total pancreas (green) and total beta cell area (red), generated by composites of low-power image scans, and high-power images of insulin-positive beta cells (yellow; DAPI in blue) for control and LepR-KO mice. Scale bars, $2 \mathrm{~mm}$ low power and $100 \mu \mathrm{m}$ high power. (c, d) Beta cell mass at 3 weeks (c) and 5 weeks (d) in control (white bars) and LepR-KO (black bars) mice. Data are means \pm SEM, 6-10 sections per pancreas, 5-7 mice per group. $* * * * p<0.0001$ vs control

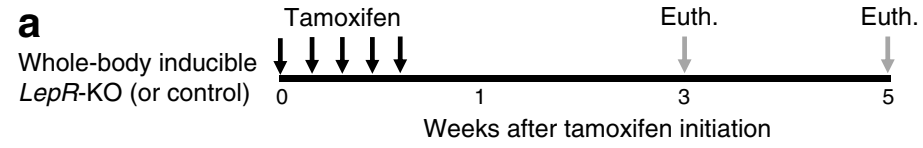

b
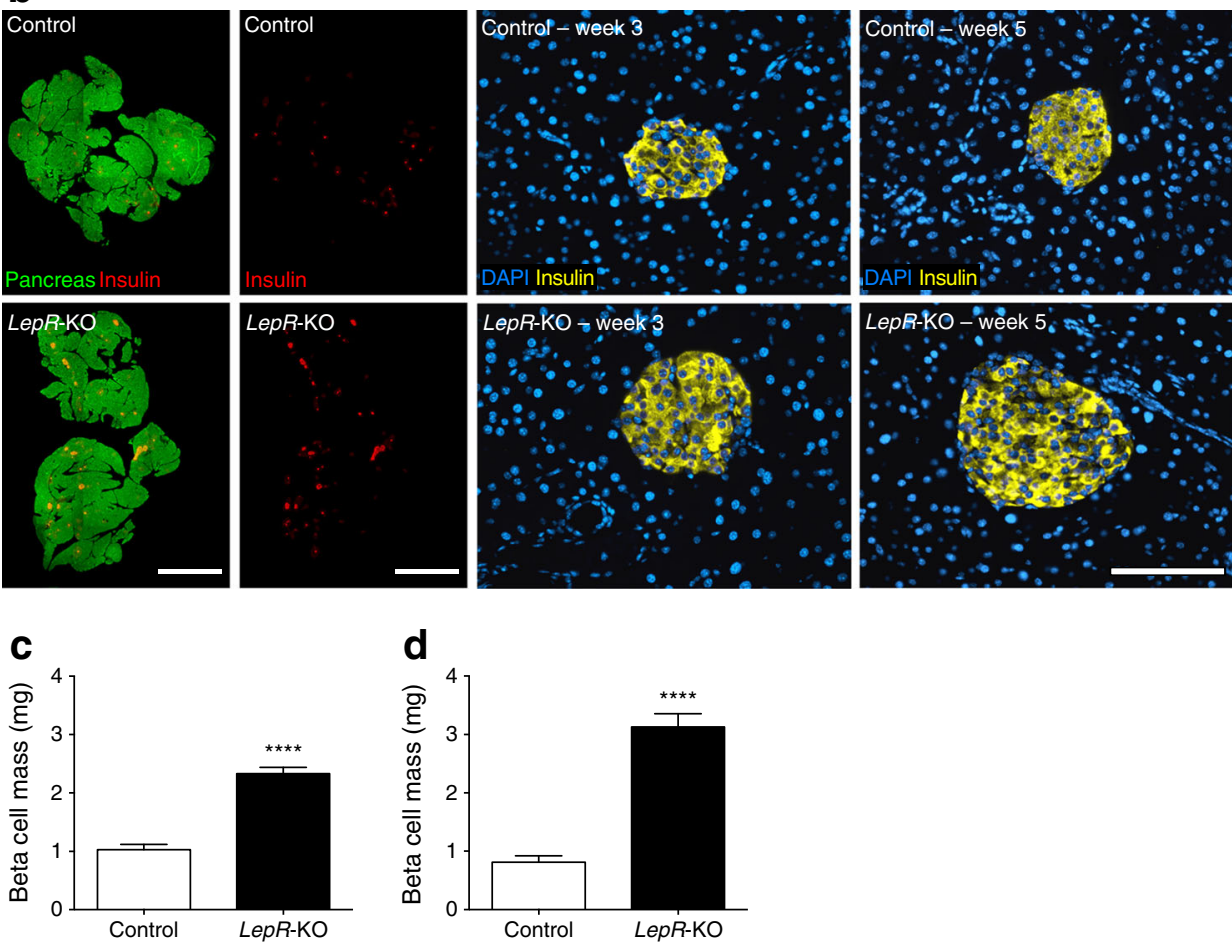

were observed relative to weeks 2-3. Thus, during 2 weeks of immense beta cell proliferation following LepR deficiency, beta cells usually divided once and rarely divided twice. This strongly supports a lineage mechanism of self-renewal (Fig. 4c) as the primary source of new beta cells.

\section{Acute LepR deficiency does not bypass the replication re-} fractory period of beta cell turnover Adult beta cell replication is governed by a 'replication refractory period', which limits beta cell turnover from occurring a second time in a recently divided beta cell (Fig. 5a) [14, 28]. However, the replication refractory period of beta cells does not appear to be permanently set, but might be altered under some conditions [14, 28]. Acute LepR deficiency-induced obesity is one of the strongest stimuli for beta cell replication, and therefore might bypass the replication refractory period (Fig. 5b). Alternatively, obesity-induced beta cell expansion could influence cell cycle progression at a later stage, without altering the refractory period (Fig. 5c). If beta cell replication is stochastic, the proportion of $\mathrm{BrdU} / \mathrm{EdU}$ co-positive beta cells should be equal to the predicted fraction of $\mathrm{BrdU} / \mathrm{EdU}$ co-positive beta cells (Fig. 5d) [14]. Alternatively, if beta cell replication is not stochastic, BrdU/EdU co-positive beta cells would be less frequent than predicted (Fig. 5d). At 3 weeks very few BrdU/EdU co-positive beta cells were observed in control mice (Fig. 5e and ESM Table 6). BrdU/EdU co-positive cells in controls represented $\sim 23 \%$ of the predicted co-positive population, substantially less than $100 \%$ (stochastic cell division) (Fig. 5f), indicating that the beta cell replication refractory period is not foreshortened [14]. BrdU/EdU co-positive beta cells were more frequent in LepR-KO mice $(2.3 \%)$, but remained considerably less than the predicted value $(21 \%$; Fig. 5e, f). This observation of extremely low numbers of double-dividing cells relative to numbers predicted persisted during weeks 4-5 (Fig. 5g, h). These results suggest that acute LepR deficiency does not shorten the beta cell replication refractory period to less than the 2 week labelling period. Therefore, the signals associated with obesity-induced beta cell expansion likely act to stimulate a pool of beta cells 'licensed' to proliferate rather than recruiting recently divided beta cells to divide again (Fig. 5c).

Beta cells do not clonally expand in acute $L e p R$ deficiency To further test for specialised progenitor cells in obesity, we used Cre loxP-based clonal analysis of beta cells. In the Brainbow2.1 model, tamoxifen induces random expression of one of four fluorescent proteins (CFP, RFP, GFP or YFP) from each allele. We crossed $U b c-C r e^{E R T 2} L e p R^{\text {loxPloxP }}$ with confetti (Rosa $\left.{ }^{(C A G-B r a i n b o w 2.1)}\right)$ to generate homozygous LepR-KO confetti mice $\left(U b c-C r e^{E R T 2} \operatorname{Rosa}^{(C A G-B r a i n b o w 2.1}{ }^{\left(e p R^{\text {loxP/loxP })}\right.}\right)$, 
a

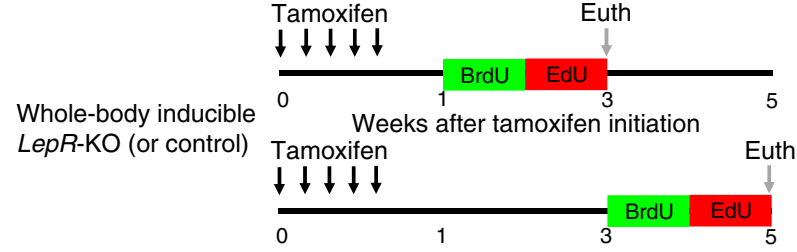

b
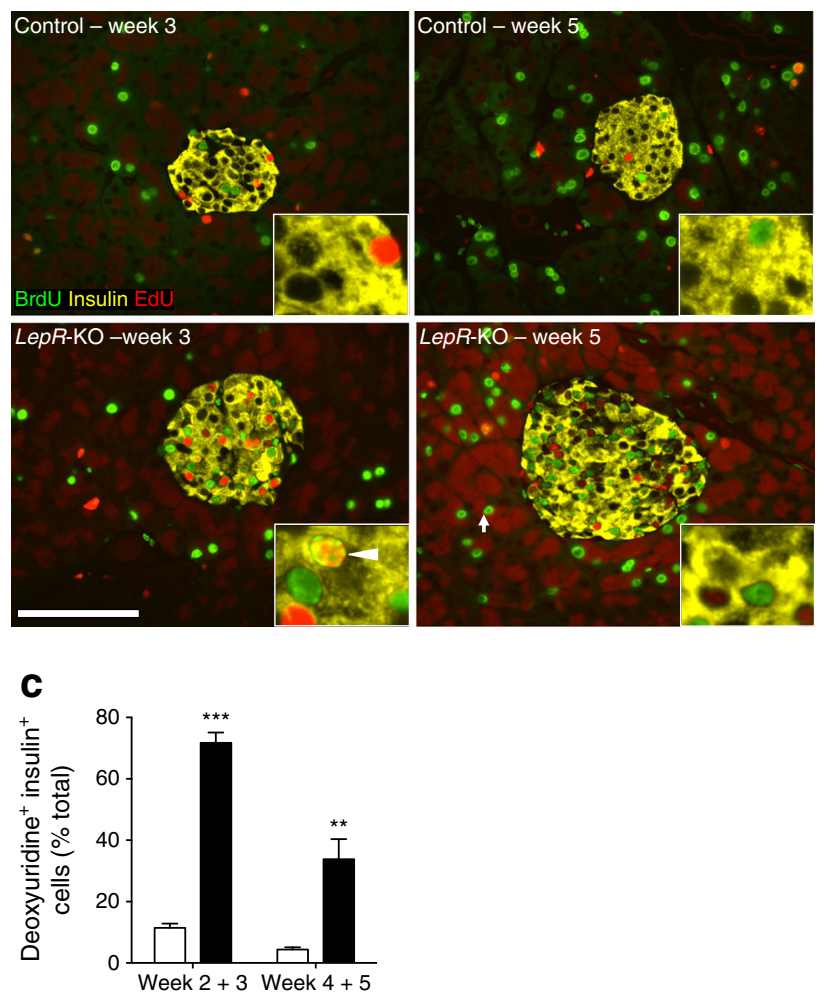

Fig. 3 Acute $L e p R$ deficiency rapidly and massively increases beta cell proliferation. (a) Timing of LepR gene deletion, thymidine analogue labelling, and euthanasia (Euth). (b) BrdU (green), insulin (yellow) and EdU (red) detection for control and LepR-KO islets. Arrowhead indicates $\mathrm{BrdU} / \mathrm{EdU}$ co-positive $\left(\mathrm{BrdU}^{+} \mathrm{EdU}^{+}\right)$beta cell. Scale bar, $100 \mu \mathrm{m}$. (c) Cumulative beta cell proliferation, measured by deoxyuridine-positive (BrdU and EdU) insulin-positive cells as a percentage of total beta cells, from control (white bars) and LepR-KO (black bars) mice. Data are means \pm SEM from $\geq 3,000$ beta cells per pancreas, $6-9$ mice per group. $* * p<0.01, * * * p<0.001$ vs control

which were treated with tamoxifen and then euthanised at various times (Fig. 6a, b and ESM Table 7). GFP and YFP were considered one distinct population represented in green (due to spectral overlap), resulting in six potential outcomes for cell labelling (CFP, RFP, GFP/YFP, CFP+RFP, $\mathrm{CFP}+\mathrm{GFP} / \mathrm{YFP}$ and $\mathrm{RFP}+\mathrm{GFP} / \mathrm{YFP}$ ) that provided high-level resolution of clones within the islet.

We hypothesised that clonal expansion by a beta cell progenitor would lead to a single dominant monochromatic clone within islets (Fig. 6b). Alternatively, beta cell expansion by self-duplication would produce islets comprised of multiple small clones of different colours. Intestinal crypts were initially multicoloured but demonstrated clonal expansion after
6 months giving rise to monochromatic villi (Fig. $6 \mathrm{c}-\mathrm{e}$ ) [27], indicating the contribution of a stem cell population to intestinal epithelia, as previously reported [27]. In contrast, pancreatic islets did not demonstrate clonal expansion, even after a 6 month washout (Fig. 6f, g and ESM Fig. 2). Labelled cells within islets were mainly found in isolation, with a few contiguous clones of between two and seven cells observed at lower frequencies than single cell clones (ESM Table 8). We then attempted to push the limits of beta cell expansion by placing LepR-KO mice on an HFD to maximise obesityinduced recruitment of new beta cells (Fig. 6h and ESM Fig. 2). These mice exhibited massive weight gain $(+41.3 \mathrm{~g})$. However, even HFD-fed LepR-KO mice did not exhibit expansion of a single dominant beta cell clone in any islet. We found no evidence of islet neogenesis in any cohort examined, as all islets surveyed were multicoloured (ESM Table 8). We conclude that acute LepR deficiency does not induce clonal expansion from a beta cell progenitor.

\section{Discussion}

Using a novel model of inducible obesity and state-of-the-art lineage tracing tools, we find that extreme metabolic demand does not alter beta cell fate. Previous attempts to induce acute

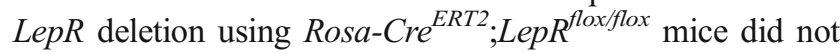
result in obesity due to inadequate floxing of $L e p R$ in the brain [29]. In contrast, $L e p R-\mathrm{KO}$ mice, via $U b c-C r e^{E R T 2} L e p R^{\text {loxPloxP }}$, doubled their body mass in 3 weeks. LepR-KO mice developed impaired glucose tolerance and insulin resistance in the absence of frank diabetes, with restoration of blood glucose through increased beta cell function and mass. Beta cell mass expansion resulted from significant beta cell proliferation without the contribution of a highly replicative progenitor cell or clonally expanding beta cells. Additionally, BrdU/EdU co-positive cells were observed in lower frequencies than predicted by chance, indicating that even during increased metabolic demand, beta cells are still restricted by the replication refractory period.

Acute LepR deficiency dramatically increased beta cell mass in response to obesity, by 3.7-fold at 5 weeks. In comparison, HFD feeding in young mice required several months to reach the magnitude of beta cell mass expansion observed in our model [19, 30, 31]. Constitutive leptin- or LepR-deficient mice (ob/ob and $d b / d b$ ) have equivalent beta cell mass to our inducible LepR-KO mice [17, 18, 32, 33]. These observations reinforce the impact of our inducible model to expand beta cells, with the ability to specifically test postnatal beta cell expansion without development of frank diabetes. Euglycaemia was maintained in LepR-KO mice for at least 6 months (ESM Table 7). Importantly, nondiabetic obese people exhibit compensatory beta cell expansion $[4,10]$. Therefore, inducible obese mice represent a compelling model with which to interrogate the signals governing mammalian beta cell expansion. 

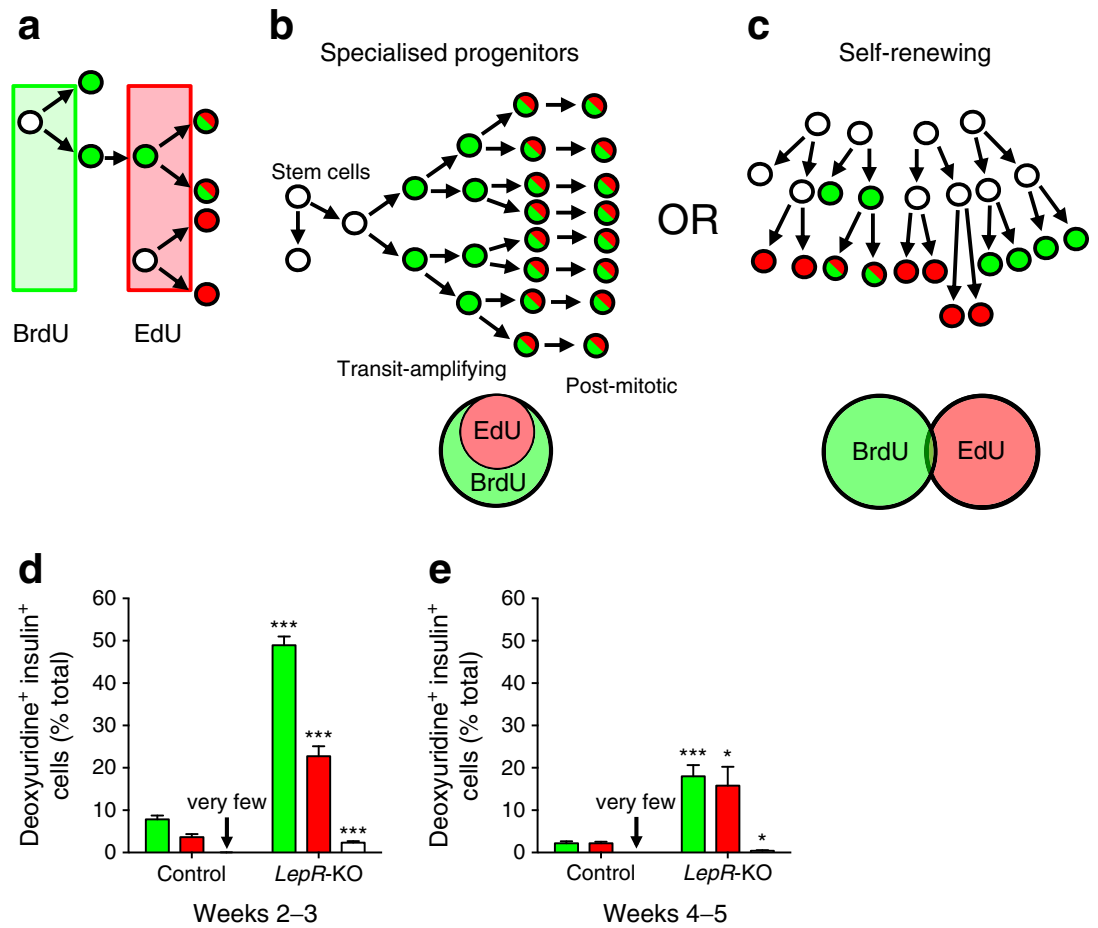

Fig. 4 Beta cells expand by self-renewal in acute LepR-deficient mice. (a) Labelling sequential cell divisions with BrdU (green) and EdU (red) results in co-labelled cells (green/red; reproduced from [14] with the permission of Elsevier). (b, c) Potential mechanisms of cell expansion employ specialised progenitors or self-renewing cell division (reproduced from [14] with permission of Elsevier). Specialised progenitor lineages

exhibit sequential cell division (b), resulting in BrdU/EdU co-labelled cells. Self-renewing lineages exhibit random cell division (c), with few co-labelled cells. (d, e) Total beta cell accumulation of BrdU (green), EdU (red) or both (white) for weeks 2-3 (d) and 4-5 (e). Data are means \pm SEM, $\geq 3,000$ beta cells per pancreas, $6-9$ mice per group. ${ }^{*} p<0.05$ and $* * * p<0.001$ vs control

Acute $L e p R$ deficiency stimulated massive beta cell proliferation, which was greatest in weeks 2 and 3, with thymidine incorporation rates of $50 \%$ ( $7.1 \%$ per day) and $23 \%$ (3.3\% per day), respectively. These observations far exceed previous results employing mitogenic stimuli such as partial pancreatectomy (PPx), streptozotocin, exendin-4, pregnancy, HFD and glucose [14, 28, 34-36]. Adult beta cell replication is strictly regulated by a replication refractory period that limits beta cell turnover from one round of the cell cycle to the next $[14,28]$. In our previous models (PPx, exendin-4, pregnancy) very few beta cells incorporated dual thymidine labels over short periods, suggesting that the refractory period is absolute and cannot be overcome by regenerative stimuli [14]. However, these studies did not include obesity, one of the most powerful stimuli for beta cell expansion. Thus, we were unable to address whether the beta cell replication refractory period is absolute or relative. Therefore, we employed the strongest tool for beta cell proliferation, using acute massive obesity to definitively test the limits of the beta cell replication refractory period. The remarkable magnitude and rapid occurrence of beta cell proliferation in LepR-KO mice was far greater than observed in previous models, hinting that obesityinduced beta cell proliferation might exceed the limitations of the refractory period. But, despite highly elevated beta cell proliferation in LepR-KO mice, acute inducible obesity did

not shorten the beta cell replication refractory period. This sharply contrasts with other studies on glucose and connective tissue growth factor, which advance potential beta cell mitogens capable of shortening the beta cell replication refractory period [28, 37]. Although LepR-KO mice were hyperglycaemic during thymidine labelling, the length of the refractory period did not decrease. A greater understanding of the mechanisms that govern the beta cell replication refractory period is important for identifying potential therapeutic targets to expand beta cells for patients with diabetes.

Acute LepR deficiency adds to the weight of evidence that beta cells expand by self-renewal. Previous studies revealed that adult beta cell regeneration largely occurs by selfduplication of pre-existing beta cells [11, 14-16]. Extensive studies have attempted to identify a stimulus that might recruit stem/progenitor cell differentiation in beta cell regeneration. Metabolic demand potently expands beta cell mass but the lineage mechanism of beta cell growth in obesity remained unknown. Researchers have speculated that stem cells potentially contribute to beta cell expansion in obesity $[10,38]$. Islet 'stem cells' would presumably involve a transit amplifying population and undergo multiple rounds of cell division incorporating both BrdU and EdU in beta cells. However, in our studies such double-labelled BrdU/EdU insulin-positive beta cells were very rare, even in response to massive obesity- 
Fig. 5 Acute LepR deficiency does not bypass the replication refractory period of beta cell turnover. (a) Adult beta cells are post-mitotic but can eventually divide more than once, limited by the replication refractory period [14]. (b, c) Opposing models of beta cell expansion suggest that increased beta cell replication results from bypassing the replication refractory period (b) or increasing cell cycle progression at later cell cycle stages (c). (d) Equation for predicted BrdU/EdU co-positive cells. (e-h) Quantification of BrdU/EdU copositive beta cells for the predicted (white) and actual (black) co-positive beta cells (\% total beta cells) (e, g) and the actual BrdU/EdU co-positive beta cells expressed as a percentage of predicted BrdU/EdU co-positive beta cells (red line) $(\mathbf{f}, \mathbf{h})$. Data are means $\pm \mathrm{SD}, \geq 3,000$ beta cells counted per pancreas, 6-9 mice per group a

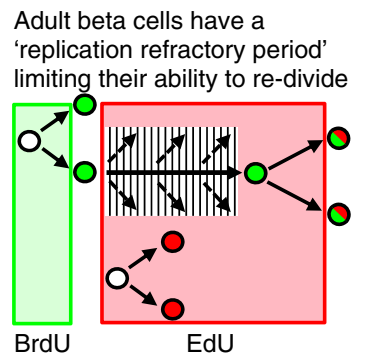

b

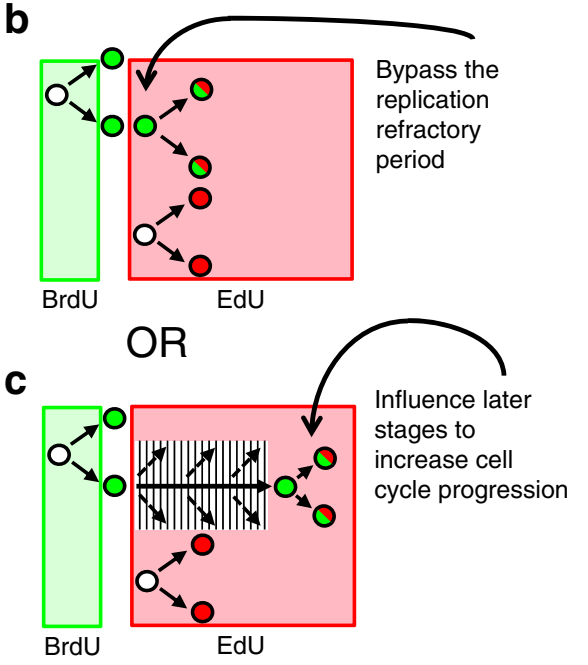

d

Predicted $\mathrm{BrdU}^{+} \mathrm{EdU}^{+}$beta cells: $\quad \frac{\text { BrdU }+ \text { beta cells }}{\text { Total beta cells }} \times \frac{\mathrm{EdU}+\text { beta cells }}{\text { Total beta cells }} \times 100 \%$

If actual < predicted, then proliferation is limited by refractory period
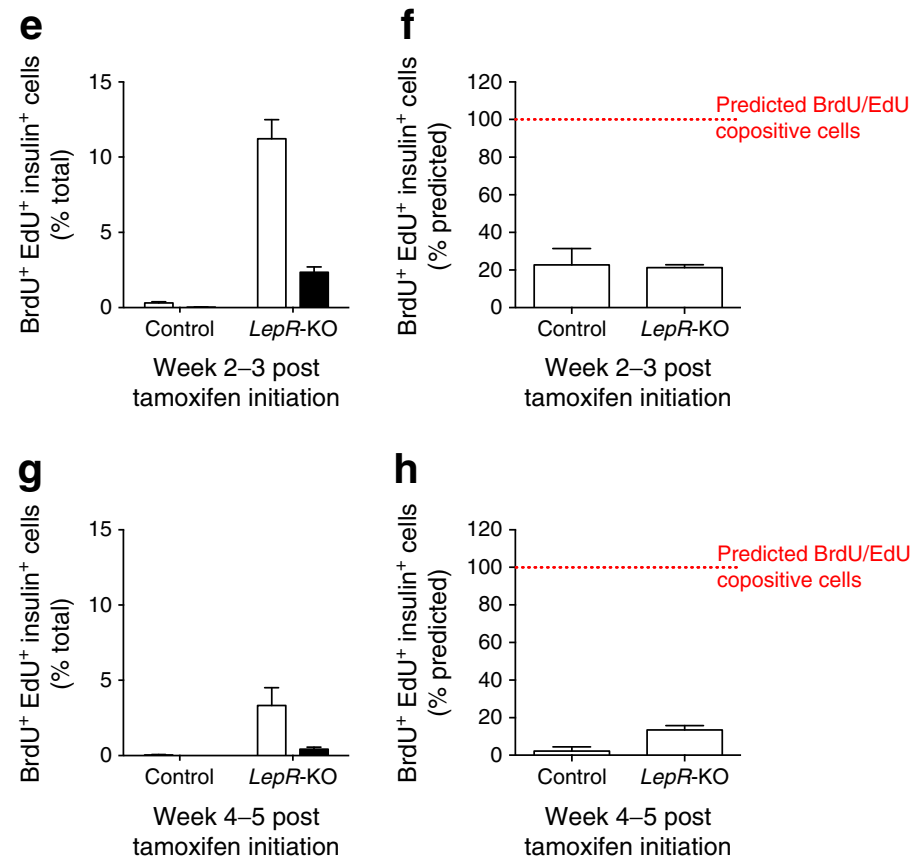

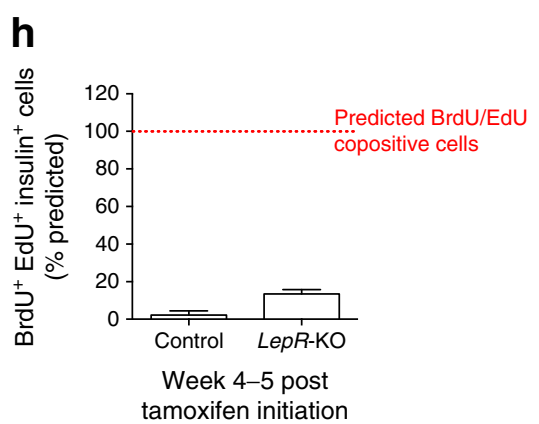

associated beta cell expansion. Although our results from short-term thymidine analogue labelling do not completely rule out contribution by a specialised progenitor to beta cell expansion, this seems highly unlikely. Taken together, our results further support the hypothesis that self-renewal of beta cells is the primary mechanism of beta cell expansion.

Elegant tools have been developed to study the clonal origins of mature differentiated cells. Brainbow2.1 has been used to examine clonal expansion of intestinal stem cells [27, 39], neuronal fate [26, 40], salivary glands [41] and cardiac growth [42]. We employed Brainbow2.1 for clonal analysis of beta cell expansion in obesity. We did not find any monochromatic islets in LepR-deficient mice, indicating that islets do not arise entirely from a common single cell at the time of labelling. Furthermore, we did not detect the presence of a single dominant clone within any islets. In contrast, we frequently found multiple small clones within individual islets. We found similar trends in LepR-KO mice challenged with an HFD. Previous reports have used low-frequency labelling of beta cells with the mosaic analysis with double markers (MADM) reporter to examine beta cell fate within rare cells $[43,44]$. These studies demonstrated that beta cell clones were 
Fig. 6 Beta cells do not clonally expand in acute LepR deficiency. (a) Timing of tamoxifen-induced recombination in $\mathrm{Ubc}-\mathrm{Cr} e^{E R T 2}$ Rosa 26(CAG-Brainbow2.1) LepR $R^{\operatorname{lox} P / o x P}$ mice. Cell labelling by Brainbow2.1 [26] was followed by 1 or 6 month washout on regular diet or HFD, and euthanasia (Euth). (b) Opposing models of beta cell expansion using the $U b c$ Cre ${ }^{\text {ERT2 Rosa26 }}$ (CAG-Brainbow2.1) $^{\text {(CA }}$ reporter: islets comprised of a large monochromatic clone indicate cells of common origin via a rapidly dividing progenitor; islets featuring single or double cell clones suggest expansion occurs through self-duplication. (c-e) Intestine from

$\mathrm{Ubc}-\mathrm{Cr} e^{E R T 2}$

Rosa26(CAG-Brainbow2.1)

LepR $R^{\text {loxP/loxP }}$ mice after 5 days (c) and 6 months (d, e), demonstrating clonal expansion in lateral (d) and transverse (e) views. (f-h) Images following 1 month (f) or 6 months (g) on a regular diet or 6 months on an HFD (h)

a

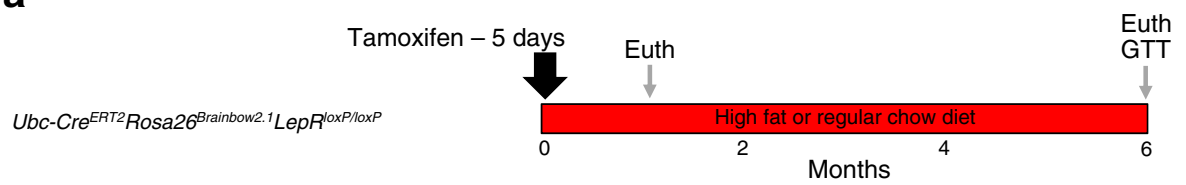

b

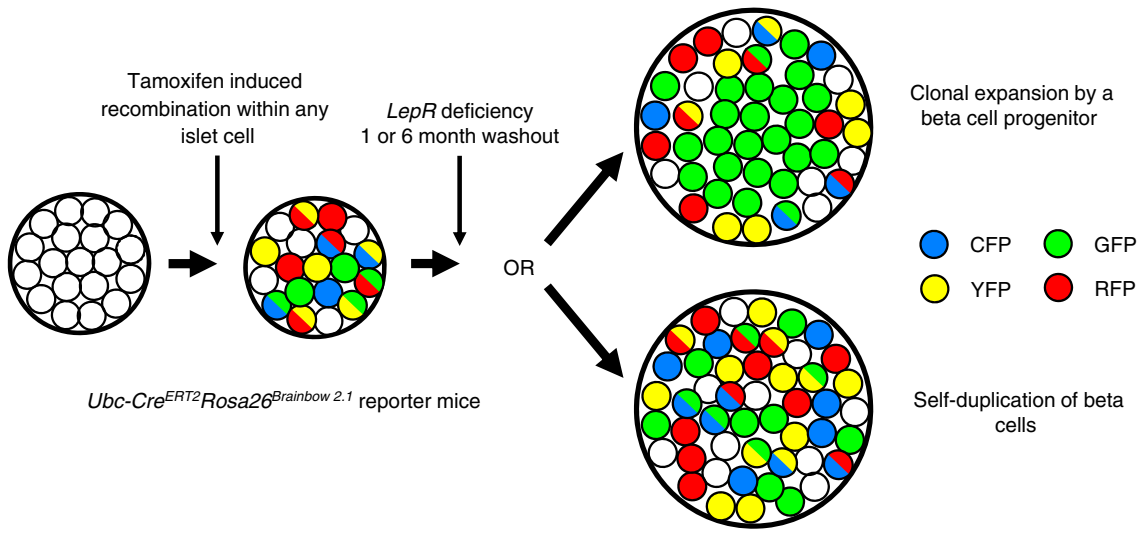

C

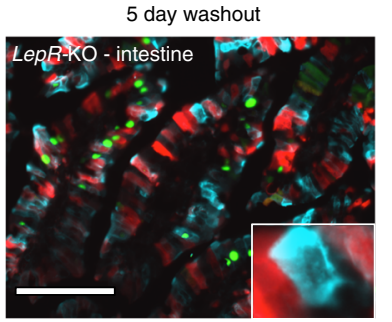

f

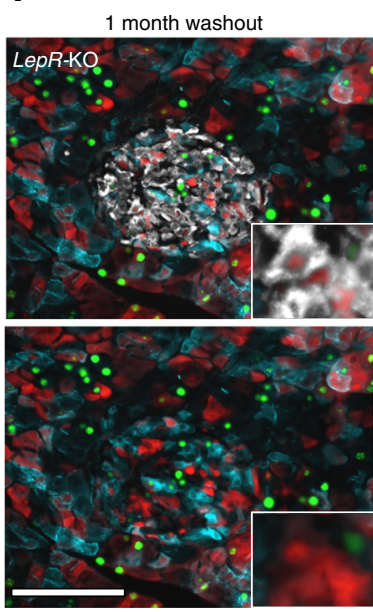

d

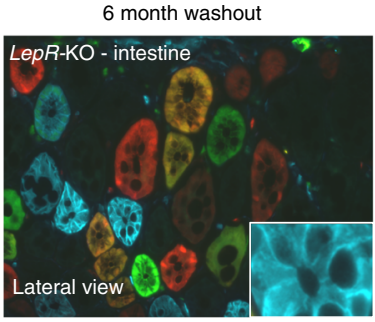

g
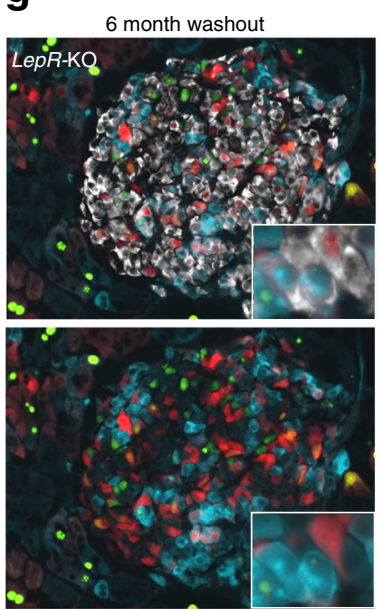

e

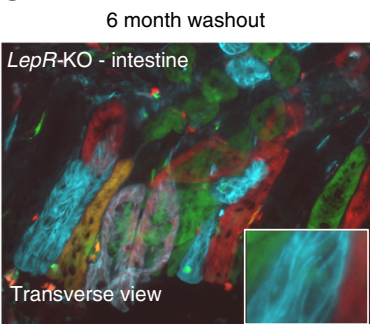

h

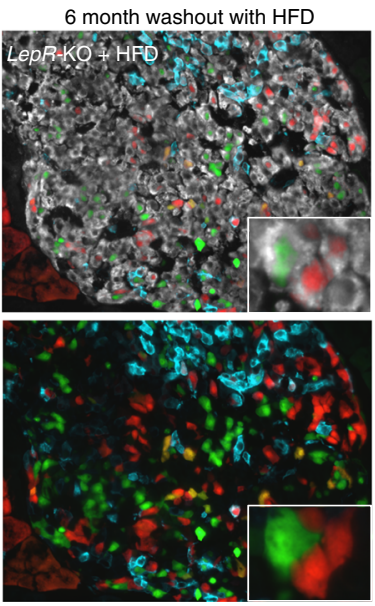

small and of similar size in normal adult beta cell growth. Our studies extend this work, showing that obesity-induced beta cell growth does not occur through clonal expansion.

The signalling mechanism(s) of beta cell expansion remain uncertain, although recent studies suggest that endoplasmic reticulum (ER) stress may regulate beta cell proliferation $[45,46]$. The increased insulin demand and subsequent hyperinsulinaemia following acute LepR deletion may stimulate ER-stress-induced beta cell proliferation [45]. Further investigation is necessary to discern the relationship between insulin demand, ER-stress and beta cell proliferation in $L e p R$-KO mice.

In summary, we developed a unique model of inducible obesity with which to determine the developmental mechanisms of obesity-associated beta cell mass expansion. Acute $L e p R$ deficiency represents the strongest stimulus for studying 
the lineage mechanisms of beta cell growth, but no evidence of a highly replicative progenitor cell or clonal expansion was found. Efforts to expand beta cells should continue to focus on identifying the signals that stimulate and regulate cell cycle progression. Moreover, acute LepR deficiency is an excellent model for future studies to interrogate the signals regulating beta cell growth for the development of potential diabetes therapies.

Acknowledgements The authors thank C. Blalock, L. Herrera, D. Kettlewell, B. Pekkattil, K. Rogers, S. Ramirez, S. Vale and C. Yee of the Texas Children's Diabetes and Endocrinology Center for their administrative expertise and support. The authors thank M. Ittmann and the Pathology and Histology Core, Baylor College of Medicine, for their expertise. The authors thank BioScience Writers for help in preparing the manuscript.

Funding The Robert and Janice McNair Foundation, NIH grant 1R01AG040110 and the Diabetes Research Center at Baylor College of Medicine (DRC - P30DK079638) provided funding. This project was supported by the Pathology and Histology Core at Baylor College of Medicine with funding from NIH grant NCI P30-CA125123. The Genetically Engineered Mouse Core at Baylor College of Medicine was funded by the Dan L. Duncan Cancer Center (assisted with transgenic mice).

Duality of interest JAK has served on the scientific advisory board of Johnson \& Johnson and currently serves on an advisory board for Lexicon. All other authors declare that there is no duality of interest associated with their contribution to this manuscript.

Contribution statement ARC conceived and designed the experiments, performed the experiments, analysed data, wrote the manuscript and approved the final version. CJL, MMR, KAK, PC and RM performed the experiments, analysed data, wrote the manuscript and approved the final version. CL designed and performed the experiments, analysed the data, wrote the manuscript and approved the final version. JAK conceived and designed the experiments, analysed data and wrote the manuscript. JAK is the guarantor of this work and has approved the final version.

Open Access This article is distributed under the terms of the Creative Commons Attribution 4.0 International License (http:// creativecommons.org/licenses/by/4.0/), which permits unrestricted use, distribution, and reproduction in any medium, provided you give appropriate credit to the original author(s) and the source, provide a link to the Creative Commons license, and indicate if changes were made.

\section{References}

1. Meier JJ, Butler AE, Saisho Y et al (2008) Beta-cell replication is the primary mechanism subserving the postnatal expansion of betacell mass in humans. Diabetes 57:1584-1594

2. Cnop M, Hughes SJ, Igoillo-Esteve M et al (2009) The long lifespan and low turnover of human islet beta cells estimated by mathematical modelling of lipofuscin accumulation. Diabetologia 53:321-330
3. Perl SKJ, Buchholz BA, Meeker AK et al (2010) Significant human $\beta$-cell turnover is limited to the first three decades of life as determined by in vivo thymidine analog incorporation and radiocarbon dating. J Clin Endocrinol Metabol 95:E234-E239

4. Saisho Y, Butler AE, Manesso E, Elashoff D, Rizza RA, Butler PC (2013) $\beta$-Cell mass and turnover in humans: effects of obesity and aging. Diabetes Care 36:111-117

5. Menge BA, Tannapfel A, Belyaev O et al (2008) Partial pancreatectomy in adult humans does not provoke $\beta$-cell regeneration. Diabetes 57:142-149

6. Meier JJ (2008) Beta cell mass in diabetes: a realistic therapeutic target? Diabetologia 51:703-713

7. Scharfmann R (2008) Expanding human beta cells. Diabetologia 51:692-693

8. Jiao Y, Le Lay J, Yu M, Naji A, Kaestner KH (2014) Elevated mouse hepatic betatrophin expression does not increase human $\beta$ cell replication in the transplant setting. Diabetes 63:1283-1288

9. Stewart AF (2014) Betatrophin versus bitter-trophin and the elephant in the room: time for a new normal in $\beta$-cell regeneration research. Diabetes 63:1198-1199

10. Butler AE, Janson J, Bonner-Weir S, Ritzel R, Rizza RA, Butler PC (2003) Beta-cell deficit and increased $\beta$-cell apoptosis in humans with type 2 diabetes. Diabetes 52:102-110

11. Dor Y, Brown J, Martinez OI, Melton DA (2004) Adult pancreatic $\beta$-cells are formed by self-duplication rather than stem-cell differentiation. Nature 429:41-46

12. Georgia S, Bhushan A (2004) Beta cell replication is the primary mechanism for maintaining postnatal beta cell mass. J Clin Invest 114:963-968

13. Teta M, Long SY, Wartschow LM, Rankin MM, Kushner JA (2005) Very slow turnover of $\beta$-cells in aged adult mice. Diabetes 54 : 2557-2567

14. Teta M, Rankin MM, Long SY, Stein GM, Kushner JA (2007) Growth and regeneration of adult beta cells does not involve specialized progenitors. Dev Cell 12:817-826

15. Kopp JL, Dubois CL, Schaffer AE et al (2011) Sox9+ ductal cells are multipotent progenitors throughout development but do not produce new endocrine cells in the normal or injured adult pancreas. Development 138:653-665

16. Rankin MM, Wilbur CJ, Rak K, Shields EJ, Granger A, Kushner JA (2013) $\beta$-Cells are not generated in pancreatic duct ligation induced injury in adult mice. Diabetes 62:1634-1645

17. Bock T, Pakkenberg B, Buschard K (2003) Increased islet volume but unchanged islet number in $o b / o b$ mice. Diabetes 52:1716-1722

18. Puff R, Dames P, Weise M et al (2011) Reduced proliferation and a high apoptotic frequency of pancreatic beta cells contribute to genetically-determined diabetes susceptibility of $\mathrm{db} / \mathrm{db}$ BKS mice. Horm Metab Res 43:306-311

19. Peyot ML, Pepin E, Lamontagne J et al (2010) Beta-cell failure in diet-induced obese mice stratified according to body weight gain: secretory dysfunction and altered islet lipid metabolism without steatosis or reduced $\beta$-cell mass. Diabetes 59:2178-2187

20. Ahren J, Ahren B, Wierup N (2010) Increased $\beta$-cell volume in mice fed a high-fat diet: a dynamic study over 12 months. Islets 2:353-356

21. Cohen P, Zhao C, Cai X et al (2001) Selective deletion of leptin receptor in neurons leads to obesity. J Clin Invest 108:1113-1121

22. Ruzankina Y, Pinzon-Guzman C, Asare A et al (2007) Deletion of the developmentally essential gene ATR in adult mice leads to agerelated phenotypes and stem cell loss. Cell Stem Cell 1:113-126

23. Tuttle AH, Rankin MM, Teta M et al (2010) Immunofluorescent detection of two thymidine analogues (CldU and IdU) in primary tissue. J Vis Exp 46, e2166

24. Li C, Najafi H, Daikhin Y et al (2003) Regulation of leucinestimulated insulin secretion and glutamine metabolism in isolated rat islets. J Biol Chem 278:2853-2858 
25. He LM, Sartori DJ, Teta M et al (2009) Cyclin D2 protein stability is regulated in pancreatic $\beta$-cells. Mol Endocrinol 23:1865-1875

26. Livet J, Weissman TA, Kang H et al (2007) Transgenic strategies for combinatorial expression of fluorescent proteins in the nervous system. Nature 450:56-62

27. Snippert HJ, van der Flier LG, Sato T et al (2010) Intestinal crypt homeostasis results from neutral competition between symmetrically dividing Lgr5 stem cells. Cell 143:134-144

28. Salpeter SJ, Klein AM, Huangfu D, Grimsby J, Dor Y (2010) Glucose and aging control the quiescence period that follows pancreatic beta cell replication. Development 137:3205-3213

29. Guo K, McMinn JE, Ludwig T et al (2007) Disruption of peripheral leptin signaling in mice results in hyperleptinemia without associated metabolic abnormalities. Endocrinology 148:3987-3997

30. Tschen SI, Dhawan S, Gurlo T, Bhushan A (2009) Age-dependent decline in $\beta$-cell proliferation restricts the capacity of $\beta$-cell regeneration in mice. Diabetes 58:1312-1320

31. Sone H, Kagawa Y (2005) Pancreatic beta cell senescence contributes to the pathogenesis of type 2 diabetes in high-fat diet-induced diabetic mice. Diabetologia 48:58-67

32. Tomita T, Doull V, Pollock HG, Krizsan D (1992) Pancreatic islets of obese hyperglycemic mice (ob/ob). Pancreas 7:367-375

33. Kawasaki F, Matsuda M, Kanda Y, Inoue H, Kaku K (2005) Structural and functional analysis of pancreatic islets preserved by pioglitazone in $\mathrm{db} / \mathrm{db}$ mice. Am J Physiol Endocrinol Metab 288: E510-E518

34. Rankin MM, Kushner JA (2009) Adaptive $\beta$-cell proliferation is severely restricted with advanced age. Diabetes 58:1365-1372

35. Alonso LC, Yokoe T, Zhang P et al (2007) Glucose infusion in mice: a new model to induce $\beta$-cell replication. Diabetes 56: 1792-1801
36. Stamateris RE, Sharma RB, Hollern DA, Alonso LC (2013) Adaptive beta-cell proliferation increases early in high-fat feeding in mice, concurrent with metabolic changes, with induction of islet cyclin D2 expression. Am J Physiol Endocrinol Metab 305:E149E159

37. Riley KG, Pasek RC, Maulis MF et al (2014) CTGF modulates adult beta-cell maturity and proliferation to promote $\beta$-cell regeneration in mice. Diabetes 64:1264-1298

38. Bates HE, Sirek A, Kiraly MA et al (2008) Adaptation to intermittent stress promotes maintenance of $\beta$-cell compensation: comparison with food restriction. Am J Physiol Endocrinol Metab 295: E947-E958

39. Koo BK, Stange DE, Sato T et al (2012) Controlled gene expression in primary Lgr5 organoid cultures. Nat Methods 9:81-83

40. Lichtman JW, Livet J, Sanes JR (2008) A technicolour approach to the connectome. Nat Rev Neurosci 9:417-422

41. Aure MH, Konieczny SF, Ovitt CE (2015) Salivary gland homeostasis is maintained through acinar cell self-duplication. Dev Cell 33:231-237

42. Gupta V, Poss KD (2012) Clonally dominant cardiomyocytes direct heart morphogenesis. Nature 484:479-484

43. Brennand K, Huangfu D, Melton D (2007) All beta cells contribute equally to islet growth and maintenance. PLoS Biol 5, e163

44. Desgraz R, Herrera PL (2009) Pancreatic neurogenin 3-expressing cells are unipotent islet precursors. Development 136:3567-3574

45. Sharma RB, O Donnell AC, Stamateris RE et al (2015) Insulin demand regulates beta cell number via the unfolded protein response. J Clin Invest 125:3831-3846

46. Szabat M, Page MM, Panzhinskiy E et al (2016) Reduced insulin production relieves endoplasmic reticulum stress and induces beta cell proliferation. Cell Metab 23:179-193 